\title{
Alanine Aminotransferase as the First Test Parameter for Wilson's Disease
}

\author{
Hisao Hayashi*1, Kazumasa Watanabe ${ }^{2}$, Ayano Inui ${ }^{3}$, Ayako Kato ${ }^{1}$, Yasuaki Tatsumi ${ }^{1}$, \\ Akihiko Okumura ${ }^{2}$, Tomoo Fujisawa ${ }^{3}$ and Koichi Kato ${ }^{1}$ \\ ${ }^{1}$ Department of Medicine, Aichi Gakuin University School of Pharmacy, Chikusa-ku, Nagoya, Japan; ${ }^{2}$ Department of \\ Gastroenterology, Kainan Hospital, Yatomi, Japan; ${ }^{3}$ Department of Pediatric Gastroenterology and Hepatology, Saiseikai \\ Yokohama Toub Hospital, Yokohama, Kanagawa, Japan
}

\begin{abstract}
Background and Aims: The liver is the first organ affected by toxic copper in the classical and severe hepatic forms of Wilson's disease (WD). Because their associated chronic liver damage is mostly asymptomatic, an intervention using a special test including serum alanine aminotransferase (ALT) activity is needed for detecting WD. Methods: Using the modified international criteria for the diagnosis of WD, 45 patients were selected from the collective databases of our institutions, and 7 infants were reviewed from the literature. Two patients had the severe hepatic form, with normoceruloplasminemia and no mutations in ATP7B. The rapid ALT change during hemolytic anemia was adjusted for a baseline. The diagnostic potential of the ALT test was assessed from the age-dependent natural course of the liver damage of WD. Results: The natural course had three stages. ALTs were still low in some infants younger than 4 years-old. They were high in all children between the ages of 4 and 8 years-old; then, they reduced to low levels in some patients over 9 years of age. The high ALT stage represents chronic active hepatitis, and the subsequent low ALT stage is due to silent cirrhosis. The hepatic copper content is a reliable but invasive test, while urinary copper secretion is an alternative, non-invasive test for copper toxicosis of WD. The serum ceruloplasmin and ATP7B analyses are subtype tests of WD. The response to anti-copper regimens is the final test result. Conclusions: ALT could be the first parameter to test to detect WD in children between the ages of 4 and 8 years. Citation of this article: Hayashi $\mathrm{H}$, Watanabe $\mathrm{K}$, Inui $A$, Kato A, Tatsumi Y, Okumura A, et al. Alanine aminotransferase as the first test parameter for Wilson's disease. J Clin Transl Hepatol 2019;7(4):293-296. doi: 10.14218/JCTH.2019.00042.
\end{abstract}

\section{Introduction}

New terminology proposed by Tatsumi et al. ${ }^{1}$ may facilitate a better understanding of the primary copper toxicosis of

Keywords: Alanine aminotransferase; $A T P 7 B$; Ceruloplasmin; Chronic active hepatitis; Wilson's disease.

Abbreviations: ALT, alanine aminotransferase; AST, aspartate aminotransferase; $\mathrm{CP}$, ceruloplasmin; WD, Wilson's disease.

Received: 5 September 2019; Revised: 25 October 2019; Accepted: 30 October 2019

*Correspondence to: Hisao Hayashi, Department of Medicine, Aichi Gakuin University School of Pharmacy, 1-100 Kusumoto-cho, Chikusa-ku, Nagoya 4648650, Japan. Tel: +81-52-757-6779, E-mail: hhayashi@dpc.agu.ac.jp
Wilson's disease (WD) and a simple approach to diagnose asymptomatic liver damage in the early stage of WD. The classical form is a primary copper-induced liver disease, which may be complicated by lenticular degeneration at a later stage, and which was initially described by SAK Wilson in $1912 .{ }^{1-3}$ In addition to increased hepatic copper of $250 \mu \mathrm{g} / \mathrm{g}$ dry liver or more, two mutant alleles in the $A T P 7 B$ gene and reduced serum levels of ceruloplasmin (CP) are found in typical cases. ${ }^{1,3}$ Other subtype tests include detection of Kayser Fleischer rings, neuropsychiatric signs, and Coombsnegative hemolytic anemia. ${ }^{1,3}$ The severe hepatic form is another copper-induced liver disease, the former name of which was idiopathic copper toxicosis, proposed by Scheinberg and Sternlieb in $1994 .^{1,4-6}$ There are currently no tests for the severe hepatic form, other than detection of increased hepatic copper and urinary copper secretion, ${ }^{1,6}$ and the new subtype tests proposed by Tatsumi et al. ${ }^{1}$ are combined results of normal CP levels and no mutations in ATP7B.

Another recommendation that Tatsumi et al. ${ }^{1}$ propose is a treatment for the copper toxicosis of WD. Every patient with WD should receive a short-term treatment trial involving anti-copper regimens, followed by life-long maintenance treatment. For the former, a poor response to anti-copper regimens indicates non-WD. Some patients with WD, especially the severe hepatic form, suddenly present with hepatic failure and require a liver transplantation without the liver biopsy essential for diagnosing WD. In such cases, the postoperative diagnosis of WD should be confirmed by the increased copper content of the removed liver.

It is now an urgent task to establish liver tests for patients with asymptomatic liver damage of WD. Aspartate aminotransferase (AST) and alanine aminotransferase (ALT) are favorable liver tests, but they are hampered by the following problems in WD patients: (1) rapid falls of AST and ALT during Coombs-negative hemolytic anemia; ${ }^{7}$ (2) natural courses of the first increasing and second decreasing stages of AST and $\mathrm{ALT}^{8}{ }^{8}$ and (3) AST having an erythrocyte fraction in WD, where it is significantly affected by copper-induced hemolysis. ${ }^{7,9}$ When the serum levels of AST and ALT are introduced as tests for the primary liver damage of WD, all problems should be overcome to minimize misdiagnosis.

In this study, we evaluated the potential of serum levels of ALT as representative of age-dependent liver disease activity of WD and determined the optimal age for the ALT test in WD patients. 
Hayashi H. et al: ALT test for Wilson's disease

Table 1. Fifty-two patients with WD and their subtypes using ATP7B analysis and serum CP levels

\begin{tabular}{|c|c|c|c|c|}
\hline Group of patients & ATP7B & $\begin{array}{l}\text { Serum CP } \\
(>20 \mathrm{mg} / \mathrm{dL})\end{array}$ & $\begin{array}{l}\text { Patient } \\
\text { number }\end{array}$ & $\begin{array}{l}\text { Major } \\
\text { subtype }\end{array}$ \\
\hline \multirow[t]{2}{*}{ Literature, $n=7$} & Two mutant alleles & Low & 7 & Classical \\
\hline & & Normal & 0 & Classical \\
\hline \multirow[t]{6}{*}{ Patient databases, $n=45$} & Two mutant alleles & Low & 38 & Classical \\
\hline & & Normal & 1 & Classical \\
\hline & One mutant allele & Low & 4 & Classical \\
\hline & & Normal & 0 & $?$ \\
\hline & No mutant allele & Low & 0 & $?$ \\
\hline & & Normal & 2 & Severe hepatic \\
\hline
\end{tabular}

Fifty-two patients with WD were subtyped using $A T P 7 B$ analysis and serum levels of CP. The presence of mutant alleles in $A T P 7 B$ and hypoceruloplasminemia less than $20 \mathrm{mg} / \mathrm{dL}$ strongly suggests the classical form of WD, while their absence suggests the severe hepatic form of WD. A total of 45 patients (7 from the literature review and 38 from the patient databases) had typical features of the classical form, while 2 patients had the severe hepatic form. Therefore, the tests, either incidental or intentional, should be adaptive for the primary liver diseases of WD consisting of the two subtypes of classical and severe hepatic forms.

Abbreviations: $C P$, ceruloplasmin; WD, Wilson's disease.

\section{Methods}

\section{Patients}

International proposals were used for the diagnosis of WD, with minor modifications. ${ }^{3}$ The original scoring system for the diagnosis consists of Kayser-Fleischer rings ( 2 points), neuropsychiatric symptoms (2), Coombs-negative hemolytic anemia (1), urinary copper (max. increase: 2 ), hepatic copper quantitative (max. increase: 2 ), serum CP level (max. decrease: 2$){ }^{10}$ and $A T P 7 B$ mutation (2 alleles: 4, one allele: 1 ). Diagnostic WD is assigned with a score of 4 or more points. Probable WD is assigned with score of 2-3 points. Unlikely WD is assigned with score of $0-1$ points. In the patients saved by liver transplantation, the copper content of the removed liver should receive a maximum of 2 points $(>250 \mu \mathrm{g} / \mathrm{g}$ dry liver weight). In patients with 2-3 points (probable WD), the diagnosis was retrospectively confirmed by giving an additional 2 points for a good response to anti-copper regimens. A removed liver with copper less than $250 \mu \mathrm{g} / \mathrm{g}$ dry liver and a poor response to anti-copper regimens were set as exclusion criteria. WD cases complicated with either chronic cholestasis or other chronic liver disease, including chronic viral hepatitis, nonalcoholic steatohepatitis, etc., were also excluded from the study.

A total of 52 patients with primary copper toxicosis of WD were enrolled in the study. Forty-five Japanese patients were selected from the databases of three institutes (Aichi Gakuin University School of Pharmacy, Kainan Hospital, and Saiseikai Yokohama Toub Hospital), and 7 infant patients were cited based on a literature review of PubMed to identify relevant papers written in English since 1993. ${ }^{11-16}$ Clinical data of two Japanese patients were analyzed twice because they were followed for years without anti-copper regimens.

Based on $A T P 7 B$ analysis and serum levels of $C P$, the primary copper toxicosis of WD in the studied patients was subtyped into two forms - classical and severe hepatic WD (Table 1 ). ${ }^{1}$ Forty-five patients had typical features of the classical form, with two mutant alleles in ATP7B and low levels of serum CP $(<20 \mathrm{mg} / \mathrm{dL})$; two other patients had the severe hepatic form, with no mutation in $A T P 7 B$ and normal levels of serum CP. Five patients had atypical classical forms, with one mutant allele of $A T P 7 B$.

\section{Methods}

The study protocol was approved by the ethics committees of 3 institutes (Aichi Gakuin University School of Pharmacy,

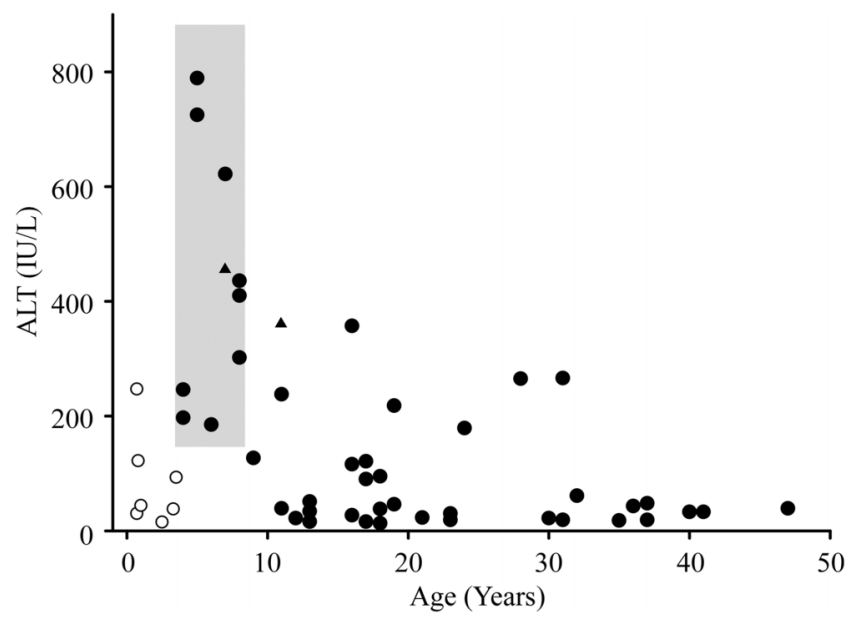

Fig. 1. Natural course of WD represented by serum ALT levels adjusted for the baseline liver disease and onset ages of patients. Rapid ALT changes during Coombs-negative hemolytic anemia of WD were adjusted to a baseline at either the prehemolytic anemia or postrecovery stage. In the patients whose WD was found after acute diseases, such as fever episodes and gastrointestinal problems, the baseline ALT was obtained at the recovery stage. Three ALT stages of WD associated with the ages of patients are shown. The first stage includes 7 infants with development of WD before 4 years old; ALT levels are low in 4 and high in 3 infants. The second stage consists of 10 patients aged between 4 and 8 years whose ALT levels are all high, over $150 \mathrm{IU} / \mathrm{L}$. The third stage includes 35 patients with a wide range of ALT levels; fifteen patients have pathologically high ALT levels, while 20 patients have near-normal levels. Especially, all 7 patients aged 35 years or older show ALT levels below $50 \mathrm{IU} / \mathrm{L}$. The ALT test is reliable for detecting WD at the high ALT stage of children aged between 4 and 8 years. ALT, alanine aminotransferase; WD, Wilson's disease. $\bullet$ : Classical form of Japanese patients; $\mathbf{\Delta}$ : Severe hepatic form (non-ATP7B) of Japanese patients; $O$ : Infants with the $A T P 7 B$ classical form cited from the international literature. ${ }^{11-16}$ 
Hayashi H. et al: ALT test for Wilson's disease

Table 2. Recommended diagnostic approach to the primary liver disorders of WD

\begin{tabular}{|c|c|c|c|}
\hline & & & WD \\
\hline & & Classical Form & Severe Hepatic Form \\
\hline 1 & Liver test & \multicolumn{2}{|c|}{ Serum ALT test: $>150 \mathrm{IU} / \mathrm{L}$ in children aged $4-8 \mathrm{yrs}$} \\
\hline \multirow[t]{2}{*}{2} & Copper tests & \multicolumn{2}{|c|}{ Hepatic copper more than $250 \mu \mathrm{g} / \mathrm{g}$ dry liver } \\
\hline & & \multicolumn{2}{|c|}{ Increased urinary copper secretion (basic and post-penicillamine) } \\
\hline \multirow[t]{2}{*}{3} & Subtype tests & Hypoceruloplasminemia & \multirow[t]{2}{*}{ Normoceruloplasminemia and no $A T P 7 B$ mutation } \\
\hline & & Mutations in $A T P 7 B$ & \\
\hline 4 & Anti-copper tests & \multicolumn{2}{|c|}{ Good responses on ALT reduction } \\
\hline
\end{tabular}

Copper-induced chronic active hepatitis of WD, either classical or severe hepatic forms, should be initially detected by an ALT test and confirmed by tests for copper toxicosis. Serum levels of ceruloplasmin and ATP7B are useful for subtyping. Patients with both the classical and severe hepatic forms should show good responses to anti-copper regimens. Abbreviation: ALT, alanine aminotransferase; WD, Wilson's disease.

Kainan Hospital, and Saiseikai Yokohama Toub Hospital). Serum levels of ALT were determined by one point at entry in all patients with WD, except for those with Coombs-negative hemolytic anemia and complicated acute diseases. The baseline ALT levels of hemolysis patients were obtained at the stage of pre-hemolytic anemia or recovery from anemia. ${ }^{7}$ In the patients whose WD was found after acute diseases, such as fever episodes and gastrointestinal problems, the baseline ALT was obtained at the recovery stage. Using the serum levels of ALT and onset ages of 52 patients aged between 0.7 and 47 years, a virtual image was made for the natural course of WD. The diagnostic potential of the age-dependent ALT levels was assessed for the primary liver damage of WD.

\section{Results}

Virtual images of the natural course of WD using serum ALT levels representative of primary liver disease activities and entry ages of patients are presented in Fig. 1. There are three ALT stages associated with the onset ages of patients. In the first stage, some infants younger than 4 years-old showed low ALT levels. In the second stage, all of the 10 children aged between 4 and 8 years showed high ALT levels ( $>150 \mathrm{IU} / \mathrm{L})$. In the third stage, patients over the age of 9 years showed a wide range of ALT levels; fifteen of thirty-five patients showed pathologically high ALT levels, while 20 patients had levels that were near-normal. Especially, all seven patients aged 35 years or older showed ALT levels below $50 \mathrm{IU} / \mathrm{L}$.

\section{Discussion}

Our observations strongly suggest that a standard liver test ALT - has the potential to detect most WD patients between the ages of 4 and 8 years-old. Serum ALT levels higher than $150 \mathrm{IU} / \mathrm{L}$ are representative of active chronic hepatitis of WD. ${ }^{17,18}$ Underlying mechanisms of the low ALT levels are different in the first and third stages. The former means that there is no active liver damage in some patients as yet, due to a small amount of toxic copper in the liver, ${ }^{8}$ while the latter involves silent cirrhosis in some patients. It is well known that, in chronic viral hepatitis, histological progression from chronic hepatitis to cirrhosis is associated with declining inflammation in the liver. Such transformation is of special importance in WD. A large amount of copper is mostly stored in hepatocyte lysosomes of cirrhosis as detoxified cuprothioneins. ${ }^{19,20}$ Age-dependent copper accumulation in the central nervous system is also associated with silent cirrhosis formation in classical WD. ${ }^{8}$ As a result, ALT tests partially lose the potential to detect copper-induced liver diseases in patients over 9 years-old.

Based on the natural disease course presented in this study, we can postulate that most patients aged over 9 years had asymptomatically experienced the high ALT stage when between the ages of 4 and 8 years-old. Therefore, children aged between 4 and 8 years may be suitable candidates for special intervention to detect asymptomatic but biochemically active liver damage of WD, as summarized in Table 2.

Increased hepatic copper and urinary copper secretion are reliable tests for the copper toxicosis of WD favored by the international proposal. ${ }^{3}$ Hypoceruloplasminemia ${ }^{1,10}$ and $A T P 7 B$ mutations ${ }^{1,3}$ strongly support the classical form. In contrast, their respective absence does not exclude WD; the negative combination of the two major subtyping tests for the classical form may be transient subtype tests for the severe hepatic form that has no definitive subtype tests, including gene analysis. ${ }^{1,6}$

A final diagnosis of WD should be confirmed in all children with WD, by anti-copper regimens. A recent study on young patients under 10 years of age revealed that zinc monotherapy reduced serum ALT levels ranging from $243 \pm$ 151 to $70 \pm 29 \mathrm{IU} / \mathrm{L}$ in 1 month. ${ }^{23}$ It is also important that copper-overload conditions secondary to chronic cholesta$\mathrm{sis}^{24}$ or chronic liver diseases other than $\mathrm{WD}^{25,26}$ could be differentiated by the short-term treatment test.

Because neuropsychiatric symptoms of classical WD appear at a late cirrhotic stage associated with low ALT levels, ${ }^{8}$ an intentional ALT test for children at the high ALT stage is prophylactic for neuropsychiatric complications. A smaller number of children with WD may have been affected by Coombs-negative hemolytic anemia before such a scheduled ALT test. ${ }^{8}$ A similar episode in a 7-year-old girl among the 10 patients in the high ALT stage was self-limiting, and she recovered from anemia without any sequelae. ALT intervention specialized to detect asymptomatic WD should be confirmed in a large number of patients.

\section{Conclusions}

There are two subtypes of classical and severe hepatic forms in the primary copper toxicosis of WD. A standard liver test of ALT is highly recommended for children between the ages of 4 and 8 years-old to detect asymptomatic WD. The selection criterion is a serum ALT level over $150 \mathrm{IU} / \mathrm{L}$. Increased hepatic 
copper is an etiological test. Serum CP level and ATP7B analyses are subtype tests. A marked reduction of ALT with anticopper regimens is diagnostic for WD.

\section{Conflict of interest}

The authors have no conflict of interests related to this publication.

\section{Author contributions}

Conception of study objective and design $(\mathrm{HH}, \mathrm{AI}, \mathrm{TF})$, collection of patient data $(\mathrm{KW}, \mathrm{AO}, \mathrm{KK})$, gene analysis $(\mathrm{AK}, \mathrm{YT})$.

\section{References}

[1] Tatsumi Y, Kato A, Kato K, Hayashi H. The interactions between iron and copper in genetic iron overload syndromes and primary copper toxicoses in Japan. Hepatol Res 2018;48:679-691. doi: 10.1111/hepr.13200.

[2] Compston A. Progressive lenticular degeneration: a familial nervous disease associated with cirrhosis of the liver, by S. A. Kinnier Wilson, (From the National Hospital, and the Laboratory of the National Hospital, Queen Square, London) Brain 1912: 34; 295-509. Brain 2009;132:1997-2001. doi: 10.1093/brain/awp193.

[3] Ferenci P, Caca K, Loudianos G, Mieli-Vergani G, Tanner S, Sternlieb I, et al. Diagnosis and phenotypic classification of Wilson disease. Liver Int 2003;23: 139-142. doi: 10.1034/j.1600-0676.2003.00824.x.

[4] Scheinberg IH, Sternlieb I. Is non-Indian childhood cirrhosis caused by excess dietary copper? Lancet 1994;344:1002-1004. doi: 10.1016/s01406736(94)91649-7.

[5] Müller T, Müller W, Feichtinger H. Idiopathic copper toxicosis. Am J Clin Nutr 1998;67:1082S-1086S. doi: 10.1093/ajcn/67.5.1082S.

[6] Fujisawa T, Inui A, Kawakami M, editors. Pediatric clinical hepatology. Tokyo: Tokyo Igakusha; 2017. (in Japanese).

[7] Hayashi H, Tatsumi $Y$, Yahata S, Hayashi H, Momose K, Isaji R, et al. Acute hepatic phenotype of Wilson disease: Clinical features of acute episodes and chronic lesions remaining in survivors. J Clin Transl Hepatol 2015;3: 239-245. doi: 10.14218/JCTH.2015.00032.

[8] Katano Y, Hayashi K, Hattori A, Tatsumi Y, Ueyama J, Wakusawa S, et al. Biochemical staging of the chronic hepatic lesions of Wilson disease. Nagoya J Med Sci 2014;76:139-148.

[9] Lippi G, Salvagno GL, Montagnana M, Brocco G, Guidi GC. Influence of hemolysis on routine clinical chemistry testing. Clin Chem Lab Med 2006; 44:311-316. doi: 10.1515/CCLM.2006.054.

[10] Scheinberg IH, Gitlin D. Deficiency of ceruloplasmin in patients with hepatolenticular degeneration (Wilson's disease). Science 1952;116:484-485. doi: 10.1126/science.116.3018.484.
[11] Shimizu N, Nakazono H, Watanabe A, Yamaguci $Y$, Hemmi H, Aoki T. Molecular diagnosis of Wilson's disease. Lancet 1997;349:1811-1812. doi: 10. 1016/S0140-6736(05)61691-1.

[12] Abuduxikuer K, Li LT, Qiu YL, Wang NL, Wang JS. Wilson disease with hepatic presentation in an eight-month-old boy. World J Gastroenterol 2015;21: 8981-8984. doi: 10.3748/wjg.v21.i29.8981.

[13] Kim JW, Kim JH, Seo JK, Ko JS, Chang JY, Yang HR, et al. Genetically confirmed Wilson disease in a 9-month old boy with elevations of aminotransferases. World J Hepatol 2013;5:156-159. doi: 10.4254/wjh.v5.i3.156.

[14] Usta J, Wehbeh A, Rida K, El-Rifai O, Estiphan TA, Majarian T, et al. Phenotype-genotype correlation in Wilson disease in a large Lebanese family: association of c.2299insC with hepatic and of p. Ala1003Thr with neurologic phenotype. PLoS One 2014;9:e109727. doi: 10.1371/journal. pone.0109727.

[15] Ohura T, Abukawa D, Shiraishi H, Yamaguchi A, Arashima S, Hiyamuta S, et al. Pilot study of screening for Wilson disease using dried blood spots obtained from children seen at outpatient clinics. J Inherit Metab Dis 1999 ; 22:74-80. doi: 10.1023/a:1005455401076.

[16] Yuan ZF, Wu W, Yu YL, Shen J, Mao SS, Gao F, et al. Novel mutations of the ATP7B gene in Han Chinese families with pre-symptomatic Wilson's disease. World J Pediatr 2015;11:255-260. doi: 10.1007/s12519-015-0031-5.

[17] Sternlieb I, Scheinberg IH. Chronic hepatitis as a first manifestation of Wilson's disease. Ann Intern Med 1972;76:59-64. doi: 10.7326/00034819-76-1-59.

[18] Stromeyer FW, Ishak KG. Histology of the liver in Wilson's disease: a study of 34 cases. Am J Clin Pathol 1980;73:12-24. doi: 10.1093/ajcp/73.1.12.

[19] Goldfischer S, Sternlieb I. Changes in the distribution of hepatic copper in relation to the progression of Wilson's disease (hepatolenticular degeneration). Am J Pathol 1968;53:883-901.

[20] Hanaichi T, Kidokoro R, Hayashi H, Sakamoto N. Electron probe X-ray analysis on human hepatocellular lysosomes with copper deposits: copper binding to a thiol-protein in lysosomes. Lab Invest 1984;51:592-597.

[21] Walshe JM. Serum 'free' copper in Wilson disease. QJM 2012;105:419-423. doi: $10.1093 /$ qjmed/hcr229.

[22] Squitti R, Ghidoni R, Simonelli I, Ivanova ID, Colabufo NA, Zuin M, et al. Copper dyshomeostasis in Wilson disease and Alzheimer's disease as shown by serum and urine copper indicators. J Trace Elem Med Biol 2018; 45:181-188. doi: 10.1016/j.jtemb.2017.11.005.

[23] Eda K, Mizuochi T, Iwama I, Inui A, Etani Y, Araki M, et al. Zinc monotherapy for young children with presymptomatic Wilson disease: A multicenter study in Japan. J Gastroenterol Hepatol 2018;33:264-269. doi: 10.1111/jgh. 13812.

[24] Ramraj R, Finegold MJ, Karpen SJ. Progressive familial intrahepatic cholestasis type 3: overlapping presentation with Wilson disease. Clin Pediatr (Phila) 2012;51:689-691. doi: 10.1177/0009922812451076.

[25] Piekuse L, Kreile M, Zarina A, Steinberga Z, Sondore V, Keiss J, et al. Association between inherited monogenic liver disorders and chronic hepatitis $C$ World J Hepatol 2014;6:92-97. doi: 10.4254/wjh.v6.i2.92.

[26] Tahir A, Malik FR, Ahmad I, Akhtar P. Aetiological factors of chronic liver disease in children. J Ayub Med Coll Abbottabad 2011;23:12-14. 